\title{
Research on Production Capacity of Manufacturing Enterprises Based on System Dynamics
}

\author{
QIAN Wei ${ }^{1, a}$ JIANG Chuankai ${ }^{1, b}$ \\ ${ }^{1}$ Hubei University of Technology, Wuhan,430068,China \\ a792079462@qq.com, b1126917814@qq.com
}

Keywords: manufacturing enterprise; production capacity; system dynamics; human resources Abstract. This paper uses the system dynamics to study the influence of human resources on the production capacity of manufacturing enterprises, draw the causal circuit diagram, construct the flow stock chart model, and uses Vensim software to simulate the model.Through the analysis of simulation results, it is determined that the human resource factors affect the production capacity of the manufacturing enterprises, and the countermeasures are put forward to find the balance between the number of layoffs and the number of temporary workers recruited to keep the number of skilled employees relatively stable to achieve higher productivity of enterprises.

\section{Introduction}

Production capacity refers to the enterprise in a certain period of time and a certain technical conditions for production inputs, and its can be transformed into the maximum possible amount of a certain type of product or service after comprehensive balance ${ }^{[1]}$. However, the production capacity of the manufacturing enterprise is restricted by many factors, so many manufacturing enterprises take the actual production as the index of the production capacity. The product manufacturing process of manufacturing enterprise is more complex, and the production of products, the use of the production and processing methods and the use of the production technology level of different manufacturing enterprises are different. But we can from human (member), materials, equipment, energy and information of these five subsystems to explore their production capacity, and the relationship between the five subsystems and the impact on the overall production capacity of manufacturing enterprises block diagram are shown in Figure 1.

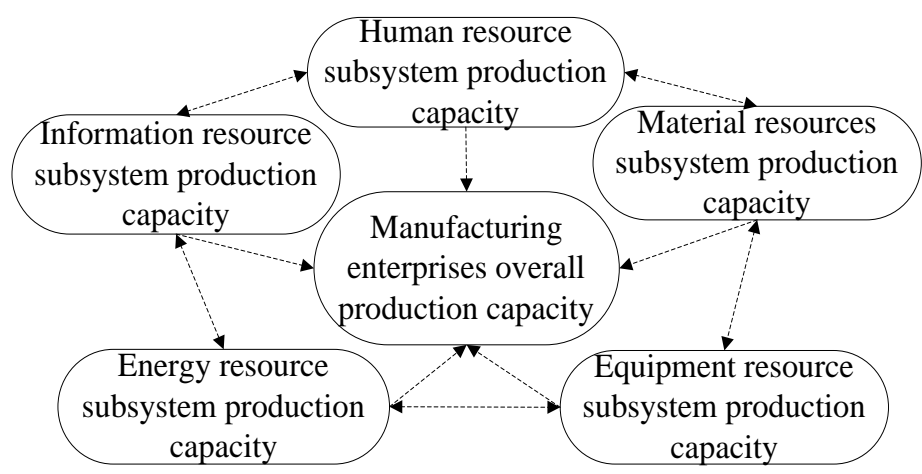

Solid line:interaction

Dotted line: enhancing effect

Fig. 1 Influence Diagram

Konosuke Matsushita, the father of Japanese management, once said: "the Panasonic Corp is not so much to manufacture product, and it is better to cultivate human ." ${ }^{\text {[2] }}$, which is the secret weapon of Japanese enterprises' success. Nowadays, many manufacturing enterprises have begun to accept this view : Human resources is the first resource of the enterprise and the source of the enterprise's life ${ }^{[3]}$, which has an indispensable influence on the enterprise's production capacity. This paper analyzes the factors involved in the production capacity of manufacturing human resources subsystem, and seeks 
to improve the production capacity of manufacturing enterprises, and provides some ideas for enterprise managers to make production decisions.

\section{Establishment of model}

System dynamics (SD) is a discipline that analyzes and studies the structure and behavior of complex information feedback. The research object is mainly for the system, examining the relationship and attributes of the elements within the system, and establishing the relevant system dynamics model.The computer simulation technology is used to simulate the model ${ }^{[4]}$, and the simulation results are used to analyze the problem.In this paper, the system dynamics Vensim PLE simulation software is used to construct and simulate the system dynamics model of the human resource subsystem of the manufacturing enterprise. The specific construction steps of the system dynamics model are shown in figure 2.

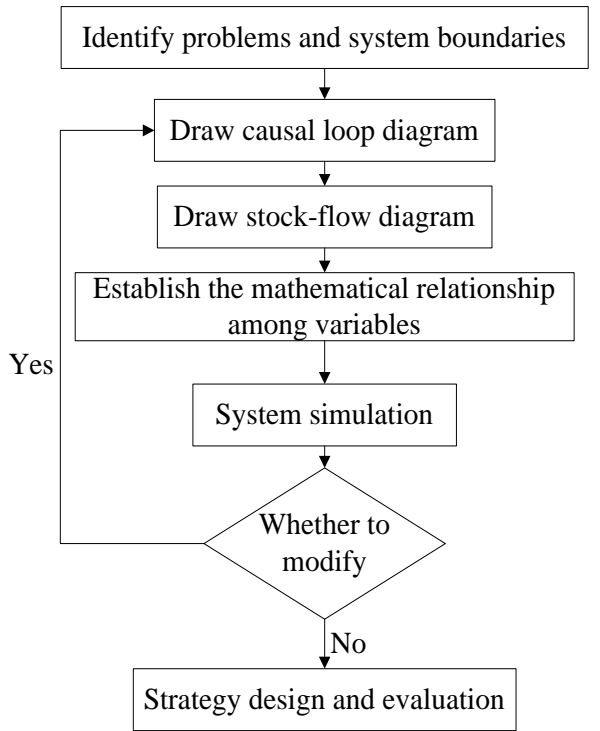

Fig. 2 Specific modeling steps

Establishment of causal loop diagram. Causal loop diagram is composed of a series of reasons and results of the closed path, and it is more vivid to show the relationship between the various elements within the system ${ }^{[5]}$. The demand of human resource in manufacturing enterprise changes with the change of customer demand. The increase of customer demand will increase the demand of employee, which will promote the expansion of recruitment plan. With the accumulation of training and practical work experience, new employees will gradually grow into skilled employees. The increase in the number of skilled employees increases the actual production of the firm, which will stimulate the demand for the entire market. In the whole system, there are new employees to grow into skilled employees, and there will be new employees and skilled employees to leave. Therefore, the number of employees has a dynamic and complex impact on the whole system. Using Vensim PLE software to draw the causal loop diagram of human resource subsystem in manufacturing enterprise are shown in Figure 3. The line-segment with the arrow in the figure are connected with elements of the system, which shows the causal relationship between the elements. The "+" and "-" symbols at the arrows are of an influential nature, and "+" indicates that the arrow pointing factor increases and decreases as the arrow source increases and decreases; "-" indicates that the factors take the opposite relationship ${ }^{[6]}$.

Establishment of stock-flow diagram. On the basis of the causal loop diagram, the stock-flow diagram is constructed, which can show the difference between the different types of variables in the system, and describe the dynamic performance of the feedback system ${ }^{[7]}$. Using Vensim PLE software to draw the stock-flow diagram of human resource subsystem in manufacturing enterprise are shown in figure 4.There are 19 variables in the stock-flow diagram. Among them, 2 state variables: number 


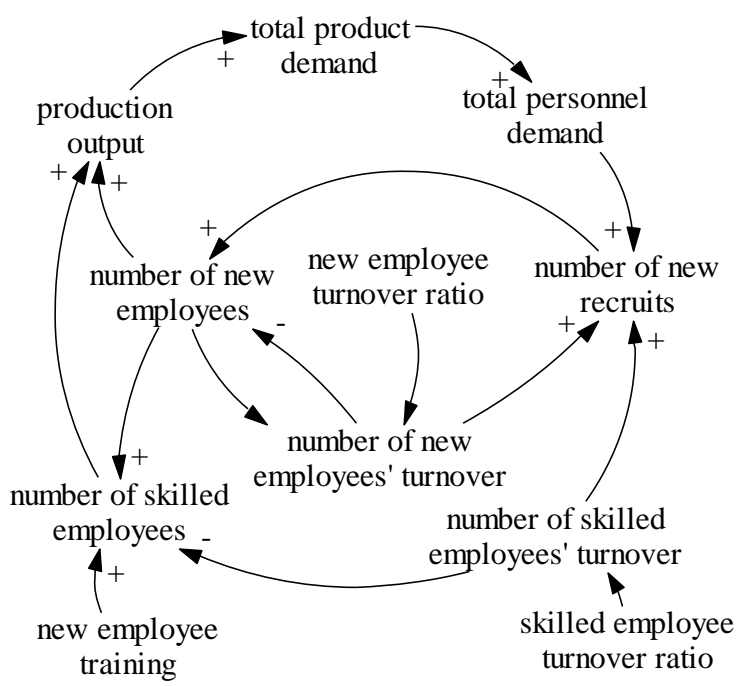

Fig. 3 Causal loop diagram

of new employees, skilled employees; 6 auxiliary variables: number of new recruits, number of new employees' turnover, number of new employees' growth, number of skilled employees' turnover, theoretical production and actual production; 8 constants: new employee turnover ratio, new employee growth ratio, skilled employee turnover ratio, labor productivity of skilled employees, labor productivity of new employees, attendance, unit production of new employees and unit production of skilled employees; 2 data: production efficiency and actual production capacity; a table function: recruitment plan.

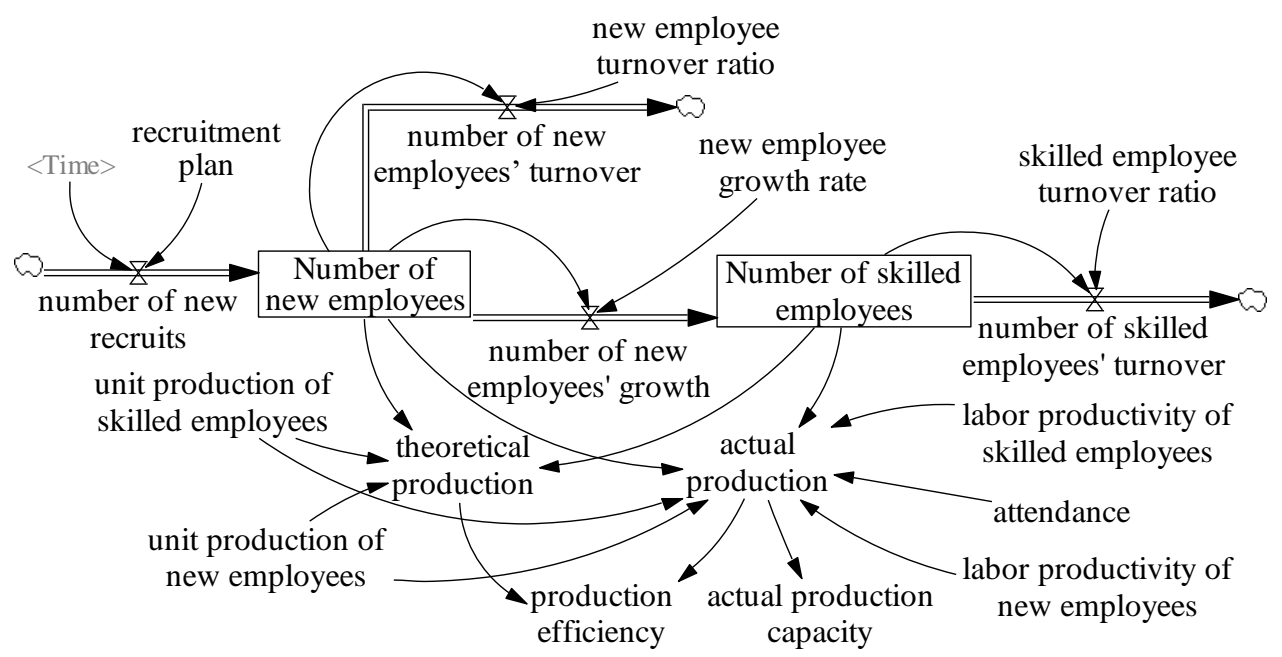

Fig. 4 Stock-flow diagram

Establishment of mathematical relationship among variables. After the definition of all the variables in the human resource subsystem of the manufacturing enterprise, the variables are independent of each other. The model at this time is only a static model. To make it a dynamic feedback structure, we should build mathematical relationship among variables. The basic mathematical relationships in the model are as follows:

(1)Number of new recruits $=$ recruitment plan $\langle$ Time $\rangle$

(2)Number of new employees' turnover $=$ number of new employees $*$ new employee turnover ratio

(3) Number of new employees' growth = number of new employees * new employee growth ratio

(4)Number of new employees = INTEGRE (number of new recruits - number of skilled employees' turnover - number of new employees' turnover, initial value)

(5)Number of skilled employees = INTEGRE (number of new employees' growth - number of 
skilled employees' turnover, initial value)

(6)Number of skilled employees' turnover $=$ number of skilled employees $*$ skilled employee turnover ratio

(7)Theoretical production=number of new employees * unit production of new employees + number of skilled employees $*$ unit production of skilled employees

(8) Actual production $=$ (number of new employees $*$ unit production of new employees* labor productivity of new employees + number of skilled employees $*$ unit production of skilled employees * labor productivity of skilled employees)* attendance

(9) Production efficiency= actual production / theoretical capacity

(10) actual production capacity $=$ actual production

\section{Simulation and analysis of the model}

Model is constructed under certain assumptions and initial conditions. Even with the same research object, the researchers cut down from different perspectives and the models are different.

Model assumptions. The theoretical model in the full sense is basically non-existent, and it is also difficult to reflect to practical problems. Reasonable assumption can simplify the model to a certain extent, and the following two assumptions are put forward for the system dynamics model:

(1) The length of new employee training period will not have an impact on the final culture results.

(2) Employees with less than 20 days of entry are regarded as new employees.

Parameter setting of system dynamics model. Before the formal simulation of the model, it is necessary to set the parameters used in the model. Normally, the setting of the parameters will appear in the constant, the initial value and the table function.

(1)Constant parameter settings. Constant parameters are generally available for enterprise data analysis and statistics obtained, and its settings are shown in Table 1:

Table 1 Constant parameter settings

\begin{tabular}{c|l|c}
\hline Major constant & Unit & Parameter values \\
\hline New employee turnover ratio & Dmnl & 0.3 \\
\hline New employee growth rate & Dmnl & 0.45 \\
\hline Skilled employee turnover ratio & Dmnl & 0.06 \\
\hline Labor productivity of skilled employees & Dmnl & 0.96 \\
\hline Labor productivity of new employees & Dmnl & 0.85 \\
\hline Attendance & Dmnl & 0.85 \\
\hline Unit production of skilled employees & Unit quantity / person & 2 \\
\hline Unit production of new employees & Unit quantity/ person & 1.3 \\
\hline
\end{tabular}

(2)Setting of initial value of variable. The state variables in the system dynamics model generally require an initial value setting. The model has two state variables (number of new employees, number of skilled employees), so there are two variables initial value settings. According to the number of existing employees, set the initial value of the variables as shown in table 2:

Table 2 Setting the initial number of employees

\begin{tabular}{l|c|c}
\hline \multicolumn{1}{c|}{ Variable name } & Unit & Parameter values \\
\hline Number of new employees & person & 25 \\
\hline Number of skilled employees & person & 100 \\
\hline
\end{tabular}

(3)Table function settings. In system dynamics, the table function is usually used to describe the change of a variable, which can reflect the nonlinear relationship between the two variables ${ }^{[8]}$. The recruitment plan in different months of the year of human resources subsystem in manufacturing enterprise is shown in the figure 5. [(0,0) - (12,30)], $(1,8),(2,2),(3,3),(4,2),(5,4),(6,30),(7,20)$, $(8,16),(9,8),(10,3),(11,27),(12,22)$. 


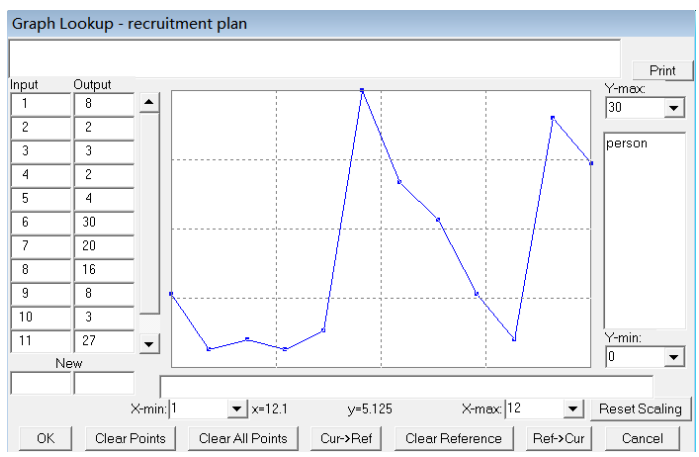

Fig. 5 Employee recruitment plan

Analysis of model simulation results. Although the establishment of the system model is relatively simple, but it can be a good response to human resources on the production capacity of manufacturing enterprises. According to the simulation results, we can get the change of the number of employees in the production line of the manufacturing enterprise and the change of the actual production quantity of the manufacturing enterprise.

(1)Trends in the number of employees. As can be seen from Figure 6, the number of new employees change relatively large. There were three peaks for the number of new employees, respectively, in January, July, and December, which in July the number of new employees reached the maximum. This is because the market demand for manufacturing enterprise has obvious low and peak seasons, the end of each year there will be a large number of staff turnover, resulting in a lot of companies will be in the beginning of the next year to invest in a certain recruitment plan.

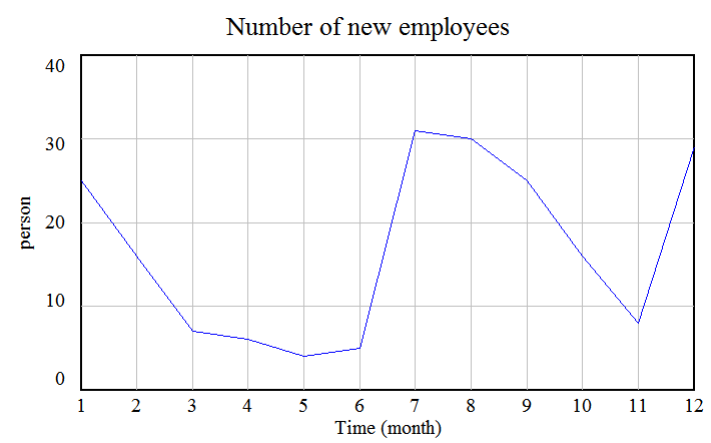

Fig.6 Changes in the number of new employees

As can be seen from Figure 7, the number of skilled employees remained stable, which is very similar to the actual situation of manufacturing enterprises. It is only when the employee leaves, that the employee will be added. The enterprise will put some recruitment plan at the beginning of each year, on the end of the year to deal with the personnel flow brings vacancy; As the particularity of the market demand forecast season and the students' summer vacation season, the enterprise will annually in July and December recruited a large number of students and staff to expand production.



Fig. 7 Changes in the number of skilled employees

(2)Changes in actual production. Combined with figure 8, it can be found that the actual production of the enterprise is determined by the number of skilled employees to a large extent, and its 
stability is about 175 units. In June, there was a trough in actual production, which is because the number of skilled employees in the enterprise declined from March to June and there were few new employees. In December, although the number of skilled employees declined slightly, but with the expansion of the enterprise recruitment plan, a large number of new employees to fill, stimulating the actual production. At the same time, due to the accumulation of training and practical experience, the new staff gradually transformed into skilled staff. And with the increase in the number of skilled workers, the actual production of enterprises increased. Thus, when there are more skilled employees in the enterprise, the actual production of the enterprise will be relatively high.

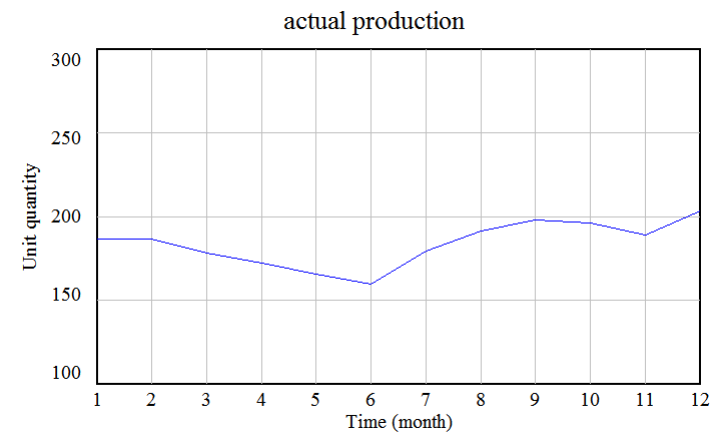

Fig. 8 Change in actual production

\section{Strategy for adjusting production capacity of enterprises}

The market demand of manufacturing enterprises has obvious low and peak seasons. At this stage, according to market demand, the enterprise staff configuration has the corresponding response measures: In the peak season demand, enterprises will temporarily expand recruitment plan, introducing some part-time students to improve production capacity and ensure higher productivity; and in the off-season demand, layoffs will be carried out. This adjustment strategy, it seems very sensible,but not otherwise. Because the students part-time more special and the production line operation is relatively simple, so pre-job training is not perfect.This is not complete training lead to students' operations are not standardized, to a certain extent affected the production capacity and efficiency.

According to the results of the model analysis, the actual production capacity is largely determined by the number of skilled employees, and the recruitment of new employees to grow into skilled employees need a certain amount of time, which will greatly affect the actual production capacity of enterprises. According to the theory of learning curve from Dr. TP Wright of Cornell University, the cumulative average working hours of the product decreases with the increase in the cumulative production of the employee's production, but the decreasing rate decreases with the increase of the cumulative production, until the unit production ability to stabilize in a range ${ }^{[9]}$. Therefore, the unit production capacity of the skilled workers and seasonal workers is very different, in the face of particularity of market demand, enterprises can not blindly expand the recruitment plan or layoffs. In light of the current market situation and its own existing production capacity, enterprises seek a balance between the number of layoffs and hiring temporary workers to maintain the number of skilled employees a relatively stable, which will increase the efficiency and reduce the additional cost of production (training costs) under the premise of ensuring production capacity.

\section{Conclusions}

This paper studies the production capacity of manufacturing enterprises from the perspective of system dynamics, constructs the human resource subsystem model of manufacturing enterprises by using its basic principles and methods, simulates the model using Vensim PLE software, analyzes influence of human resources on the production capacity of manufacturing enterprises.

Through simulation study, it is indicated that the actual production capacity of the manufacturing 
enterprise is largely determined by the skilled employees. When there are more skilled employees in the enterprise, the actual production capacity of the enterprise will be higher, which provides a theoretical basis for business managers to seek the balance between the number of hiring temporary workers and layoffs in response to low and peak seasons of market demand.

\section{Reference}

[1] Huaizu Wang: Production Planning and Control (China Science and Technology Publishing House, China 2005), p.117.

[2] Xianlu Du: The oriental culture, Vol.12(2012), p.34-36.(In Chinese)

[3] Ching Wang: China market,Vol.13(2014), p.53-54. (In Chinese)

[4] Yongguang Zhong, Xiaojing Jia , Ying Qian: System dynamics (Second Edition) (Science Press, China 2013), p.3.

[5] Miao Li: Development and evaluation of higher education, 2013 No.29, p.18-22. (In Chinese)

[6] Fu Wang: Application of system engineering in distribution center logistics system (MS., Xiamen University, China 2006), p.18-19.

[7] Yajing Zhao: Commodity and quality: theoretical research,Vol.8 (2011), p. 80-81.(In Chinese)

[8] Haiyan Wang: Research on production efficiency improvement of manufacturing enterprises based on system dynamics (MS., Chengdu University of Technology, China 2013), p.33.

[9] Shuping Yi , Fu Guo: Basic industrial engineering (Machinery Industry Press, China 2013), p.326.

[10] Weihong Xie, Yongjian Wang, Luan Jiang, Guanyu Zhang:International Forum on Information Technology and Applications (Kunming, China, July16-18, 2010).Vol.3, p.373-376.

[11] Jefferson G H, BAIH, GUANX, et al:Economics of Innovation and New Technology,Vol.15(2006), No.5, p.345-366.

[12] Xia Wang: Management and technology of small and medium sized enterprises,2009 No.36, p.287-288.(In Chinese)

[13] Dahui Bo, Lijuan Kong, Xuefeng Xu: Journal of Qingdao University (NATURAL SCIENCE EDITION), Vol.22 (2009), No.3, p.71-77.(In Chinese)

[14] Guoying Wang: Journal of Wuhan Institute of Metallurgy Administrators, Vol.25(2015),No.4, p.14-16.(In Chinese) 